\title{
Long-range ground deformation monitoring by InSAR analysis
}

\author{
S. Rokugawa and T. Nakamura \\ The University of Tokyo, Tokyo, Japan \\ Correspondence to: T. Nakamura (t-nakamura@tmi.t.u-tokyo.ac.jp) \\ Published: 12 November 2015
}

\begin{abstract}
InSAR (Interferometric Synthetic Aperture Radar) analysis is an effective technique to map 3dimensional surface deformation with high spatial resolution. The aim of this study was to evaluate the capability of InSAR analysis when applied to ground monitoring of an environmental disaster. We performed a time series InSAR analysis using ENVISAT/ASAR and ALOS/PALSAR data and commercial software to investigate subsidence around the Kanto District of Japan. We also investigated techniques for efficient early detection of landslides in Kyushu using time series analysis that incorporated synthetic aperture radar (SAR) images. ENVISAT/ASAR data acquired from 2003-2010 and ALOS/PALSAR data acquired from 2006-2011 were used to detect poorly expressed geomorphological deformation by conducting time series analyses of periodically acquired SAR data. In addition, to remove noise caused by geographical feature stripes or phase retardation, we applied median filtering, histogram extraction processing, and clarification of the displacement with a Laplacian filter. The main functions of the InSAR time series analysis are the calculation of phase differences between two images and the inversion with smoothness constraint for the estimation of deformation along the line of sight. The results enabled us to establish criteria for the selection of suitable InSAR data pairs, and provided the final error estimation of the derived surface deformation. The results of the analysis in the Kanto District suggested that localized areas of uplift and subsidence have occurred at irregular intervals in this area. Furthermore, the method offers the possibility of early warning of environmental disasters such as landslide and abrupt subsidence. Our results confirm the effectiveness of InSAR analysis for the monitoring of ground deformation over wide areas via the detection of localized subsidence and landslides.
\end{abstract}

\section{Introduction}

Recently, large-scale typhoons and unusual weather have increased local heavy rain, producing conditions that are prone to large-scale landslides and causing extensive damage, because of the steep topography of many mountainous regions in Japan. Therefore, it is essential to develop techniques to detect such landslides before their occurrence to plan countermeasure. In the present study, we demonstrate the use of InSAR technique in detecting early signs of landslides and discuss about the production of related hazard maps. Firstly, we applied the method of measuring longrange ground deformation by combining InSAR and timeseries analysis (Ferretti et al., 2000, 2001; Berardino et al., 2002; Deguchi et al., 2009) in the Kanto Plain, Japan, using 54 ENVISAT/ASAR images. The Kanto Plain started to subside in the 1910s, with the main causes of land subsidence related to the intake of ground-water for the use in industries, agriculture, waterworks, and other fields. In the Kujukuri area, the exploitation of soluble natural gas also counts. A subsidence of approximately $2 \mathrm{~cm}$ per year was detected. Then, in Kyushu area, Mt. Wanitsuka and Tsukahara dam in Miyazaki prefecture, and Imari area of Saga prefecture, interference fringes were found at the uncollapsed areas.

\section{Method}

InSAR is the most appropriate technique to investigate ground movements over large areas. This monitoring technique can offer many advantages, particularly in the detection of the precursory phenomena of landslide disasters. We 
conducted an investigation into landslide disasters occurring between 2008 and 2009 in the region around the Wanitsuka Mountains. We used a SAR PALSAR stack of 10 scenes acquired from December 2006 to April 2011. In the Imari area, northern Kyushu, we used 38 SAR interference images acquired between May 2006 and January 2011. Near the Tsukahara dam in the Mimikawa upstream region, Miyazaki prefecture, we used 22 scenes acquired in descending mode between February 2007 and April 2011 and 13 ascending scenes acquired from October 2007 and December 2010. Then, we quantitatively evaluated the reliability of the InSAR data and conducted time series analysis to clarify variations in local upheaval or settlement in order to assess ground deformation.

All the areas addressed by this study can be considered prone to landslide disaster. However, the InSAR picture that was obtained from these areas was unclear, making it an ideal location to test our newly developed detection technique. Therefore, we extracted information for a landslide by applying filters to remove noise and clarify ground movement. As a result, any place other than the point where landslide happened, I was able to detect the point considered that an initial change was caused. During this process, we corrected for the measurement error associated with InSAR and assessed the effectiveness of the technique. However, it proved difficult to remove all noise. A composite filter incorporating three individual filtering techniques has been used.

\subsection{Median filtering}

We calculated a median using a $3 \times 3$ area comprising a target pixel and its neighbouring pixels; the value is obtained as object pixel value. This filter was shown to be acceptable for the removal of spot-formed noise in InSAR images.

\subsection{Histogram extraction processing}

We normalised the InSAR displacement data and removed all small displacements from the SAR image. I removed $80 \%$ of low order by the ground displacement magnitude detection of the $\mathrm{cm}$ unit and, in the case of $\mathrm{mm}$ unit, removed low order $60 \%$.

\subsection{Laplacian filter}

We used a $51 \times 51$ operator derived from a four-dimensional operator that radicalised the surface displacement using a standard second derivative filter to produce a weighted-mean picture element area.

\section{Result and discussion}

Maps with the location of the investigated sites and the results of InSAR analysis are shown in Fig. 1 (Geological Survey of Japan, 2010). Figure 2 illustrates the interferogram

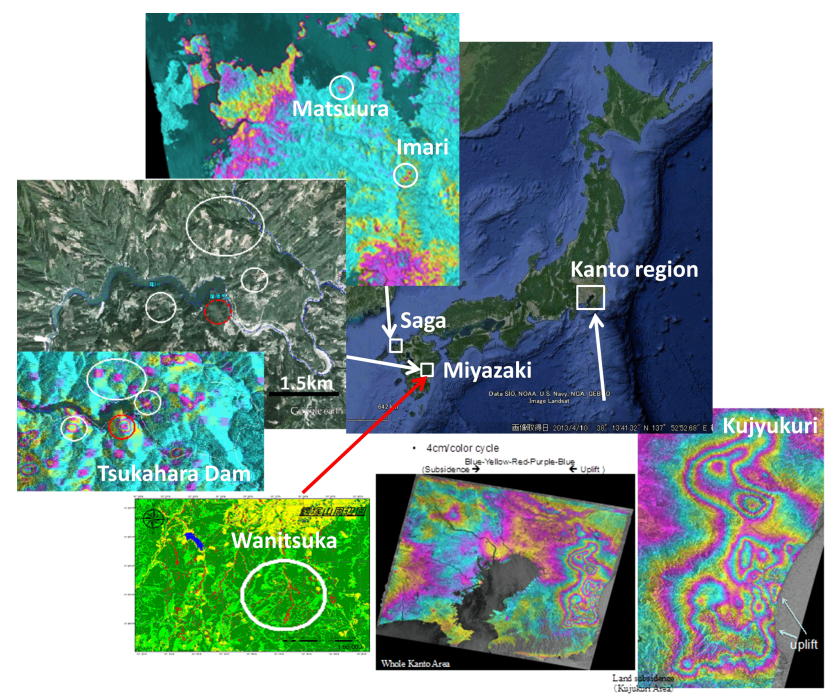

Figure 1. Map of study area and results of InSAR analysis.
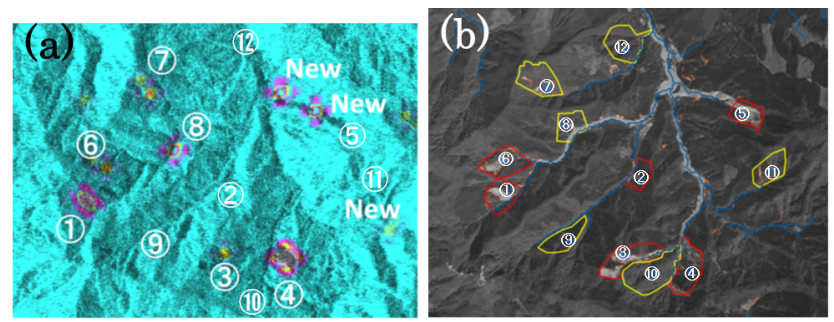

Figure 2. (a) The interferogram around the Wanitsuka Mountains in the Miyazaki Prefecture (ALOS/PALSAR data acquired from 2006 to 2011). (b) Collapsed area (red) and uncollapsed area (yellow) was interpreted based on aerial photographs acquired from 1946 to 2005 .

for the region around the Wanitsuka Mountains and an interpretation of the collapsed area based on aerial photographs, which confirms the changes in the ground movement suggested by previous field investigation. On the basis of the InSAR results, we believe that fluctuations in the ground surface continued after collapse. Collapse induced by the typhoon of 2005 was not detected in area 7-12, although evidence suggests that subsidence continued in these areas following the typhoon. A subsidence of approximately $9 \mathrm{~cm}$ was found in area 4 over a period of two years and eight months; this represents the greatest change in ground elevation detected in the region, although subsidence of $4-6 \mathrm{~cm}$ was found in other areas. These results suggest that the technique is suitable for the assessment of such ground movements. However, problems remain in terms of the interpretation of the consequence of the research technique by the application to more areas and evaluation of the application limit. Therefore, the technique has been verified through further analysis. Figures 3 and 4 illustrate the results obtained for an InSAR analysis of the Imari area using various fil- 


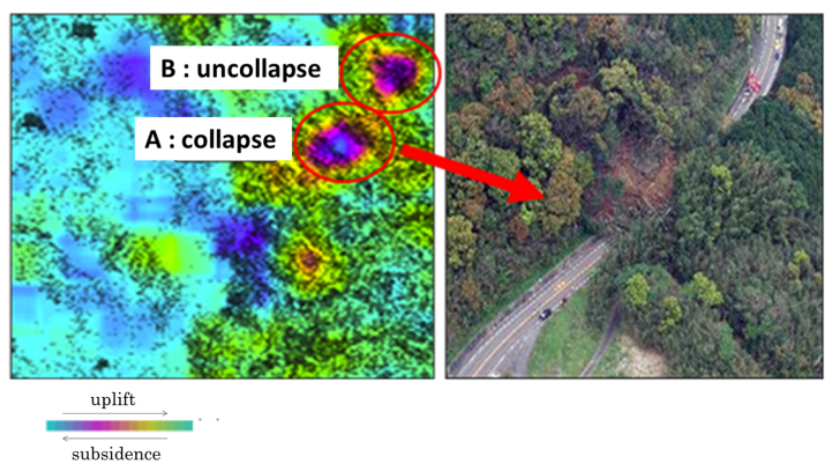

Figure 3. Imari area collapsed point (A) and uncollapsed point (B) were extracted based on InSAR analysis (left image). Actual spot of the collapse (right photo).
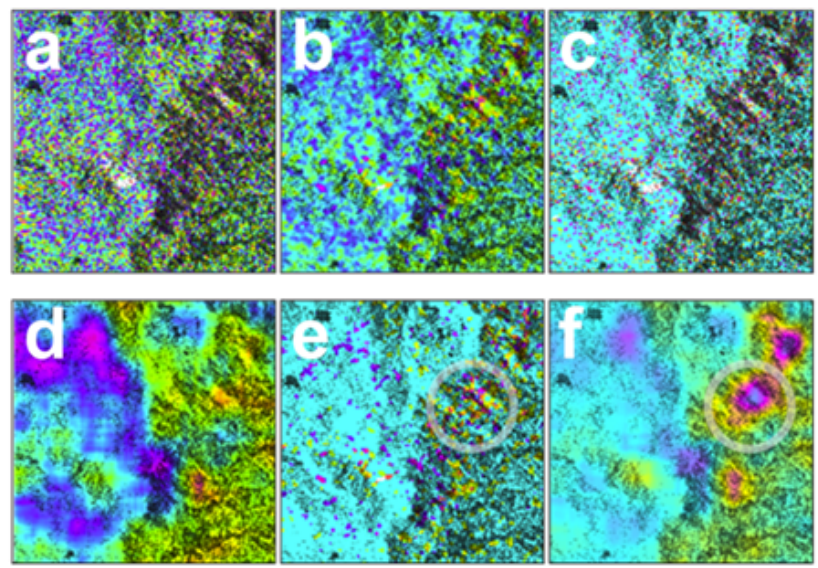

Figure 4. Imari area: effects of filter application to InSAR outcome for the Imari area. Circles indicate areas of landsliding. (a) InSAR result, (b) median filtering, (c) histogram extraction (upper $20 \%$ extract of the normalised displacement), (d) Laplacian filter, (e) median filtering and histogram extraction, (f) median filtering and histogram extraction and Laplacian filter.

ter processes. Landslide inception is difficult to be detected based on the outcome provided in Fig. $4 \mathrm{a}$ and c, because big noise has not been removed from these images. Conversely, the representation of surface displacements is improved in Fig. 4b, from which big noise (but not small noise) has been removed. Figure $4 \mathrm{~d}$ illustrates the results obtained by emphasizing displacement and noise; surface displacements can be clearly detected in this image, indicating that the use of a Laplacian filtering is effective for noise removal. Figure $4 \mathrm{e}$ illustrates the results achieved by applying both median filtering and histogram extraction and suggests that noise reduction is useful in the acquisition of displacement magnitude. The location of the landslide is clearly indicated in Fig. 4f, for which all filtering techniques were applied. The results presented here indicate that InSAR is effective in detecting microdisplacements that occur during the early stages of landsliding and that detection of these displacements can

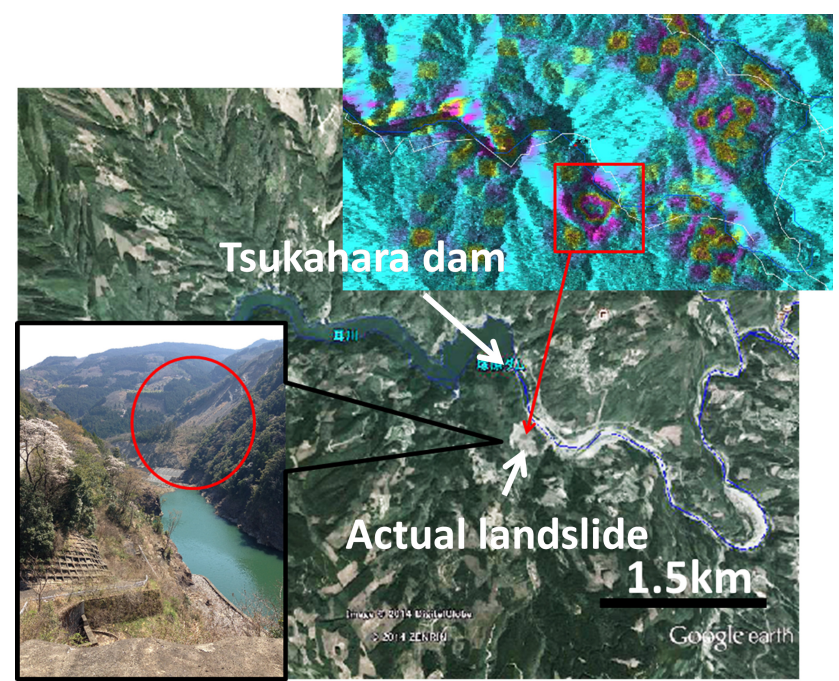

Figure 5. Collapse to the south of Tsukahara dam was extracted from ascending PALSAR image. (Base map is from Google earth)

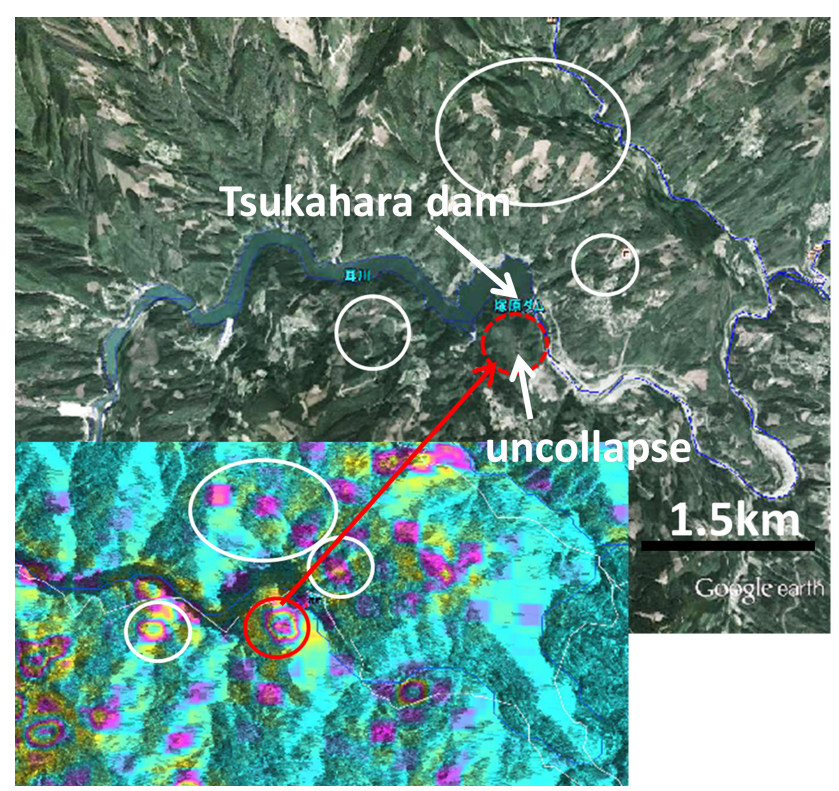

Figure 6. Uncollapsed zone to the south of Tsukahara dam was extracted from descending PALSAR image. (Base map is from Google earth)

be improved by application of filtering processes, at least in the Imari area. However, a point considered to have initial fluctuation other than the point where landslide happened has been detected as highlighted in Figs. 3b and 4f. Finally, different displacements were detected depending on the acquisition more of microwave radiation near the Tsukahara dam in the Mimikawa upstream region (Figs. 5 and 6). Collapse at the south of Tsukahara dam was extracted from ascending PALSAR image, as shown in Fig. 5. On the other hand, an uncollapsed area at the south of Tsukahara dam was ex- 
tracted from descending PALSAR image, as shown in Fig. 6. In spite of an uncollapsed point, strong interference fringes are seen and it seems that it has a high possibility that collapse will take place in the near future.

\section{Conclusions}

The result of InSAR analysis shows that the position of slope collapse can be detected with high precision. Moreover, detection of the parts where the slope collapse is expected to occur in the near future is also possible. The results here presented suggest that the proposed technique can offer information useful to build up a first ground movement system for landslide detection, and then in deciding where monitoring components such as GPS equipment should be placed. We test the effectiveness of the measures described here by performing in-situ surveys in the critical zones pointed out by InSAR. The results confirm that our techniques are effective in detecting landslide-related ground displacement over wide areas. Furthermore, we have demonstrated that it is possible to construct hazard maps for large areas based on the detected ground movements. Therefore, InSAR processing may make a considerable contribution to disaster prevention.

Acknowledgements. The authors would like to thank ERSDAC for providing PALSAR L-band data.

\section{References}

Berardino, P., Fornaro, G., Lanari, R., and Sansosti, E.: A New Algorithm for Surface Deformation Monitoring Based on Small Baseline Differential SAR Interferograms, IEEE Trans. Geosci. Remote, 40, 2375-2383, 2002.

Deguchi, T., Rokugawa, S., and Matsushima: Long-term ground deformation measurement by time series analysis for SAR interferometry, Journal of the Remote Sensing Society of Japan, 29, 418-428, 2009.

Ferretti, A., Prati, C., and Rocca, F.: Nonlinear subsidence rate estimation using permanent scatters in differential SAR interferometry, IEEE T. Geosci. Remote, 38, 2202-2212, 2000.

Ferretti, A., Prati, C., and Rocca, F.: Permanent scatterers in SAR Interferometry, IEEE Trans. Geosci. Remote, 39, 8-20, 2001.

Geological Survey of Japan: AIST, Seamless Digital Geological Map of Japan (1:200,000), 2010. 